\title{
Foster parent experiment reveals no genotype-environment correlation in the external morphology of Ficedula albicollis, the collared flycatcher
}

\author{
LARS GUSTAFSSON* \& JUHA MERILÄ \\ Department of Zoology, Uppsala University, Villavägen 9, S-752 36 Uppsala, Sweden
}

\begin{abstract}
Heritabilities of quantitative traits have recently been estimated in several natural populations of birds. The predictive value of these estimates is based on the assumption that heritability estimates are not inflated by an environmental correlation between parent and offspring. We here show with a foster experiment that heritability estimates of seven morphological traits in the collared flycatcher are not inflated as a result of a genotype-environment correlation. Surprisingly, there was a negative foster parent-offspring relation in a poor environment. It is suggested that large foster parents have more difficulties raising offspring under poor environmental conditions than do small foster parents. Our experiment also provides evidence for a heritable basis of phenotypic variation in feather traits and that the morphology of the collared flycatcher could respond rapidly to selection.
\end{abstract}

Keywords: Ficedula albicollis, foster experiment, genotype-environment correlation, heritability, quantitative traits.

\section{Introduction}

Heritabilities of quantitative traits have been recently estimated in several natural populations of birds (Boag \& van Noordwijk, 1987; Larsson \& Forslund, 1992; Merilä \& Gustafsson, 1993). As heritability measures reflect the degree to which variation of a trait is under genetic influence (Falconer, 1989), the moderate to high heritabilities reported in these studies suggest ample opportunity for further evolution in avian morphology. However, the predictive value of these estimates depends greatly on the particular circumstances under which they are measured (van Noordwijk et al., 1988; Alatalo et al., 1990; Rauscher, 1992) and on the assumption that heritability estimates are not inflated as a result of environmental correlation between offspring and parents (Turner \& Young, 1969; Feldman \& Lewontin, 1975; Falconer, 1989). Environmental correlation among relatives arises from different frequency distributions of genotypes over environments (Falconer, 1989; Stearns, 1992). For example, if the environments vary greatly among different families within a population and offspring are inclined to return to their natal habitats as adults, parents and offspring will tend to resemble each other

${ }^{*}$ Correspondence. for environmental reasons more than do members of the population taken at random (Larsson \& Forslund, 1992). As a consequence, the heritabilities estimated from parent-offspring regressions may be misleading. Also, genotype-by-environment interactions $(\mathrm{G} \times \mathrm{E})$ can be estimated only if there are no genotypeenvironment correlations, which will inevitably confound measures of $\mathrm{G} \times \mathrm{E}$ interactions (Stearns, 1992).

Although non-genetic causes of resemblance between offspring and parents have been documented in a few cases (James, 1983; James \& NeSmith, 1988; Larsson \& Forslund, 1992; Rhymer, 1992), few studies have been carried out where controlled experiments have been set up to evaluate directly the environmental contribution to parent-offspring resemblance (Smith \& Dhondt, 1980; Dhondt, 1982; Alatalo \& Lundberg, 1986; Alatalo \& Gustafsson, 1988; Wiggins, 1989). Most of these studies have focused on only one trait and surprisingly few have dealt with plumage traits. As a matter of fact, no experimental confirmation exists of the heritability of such traits.

We present here results of a foster parent experiment focusing on whether the heritability estimates of seven external morphological traits of the collared flycatcher (Ficedula albicollis) are inflated due to genotype-environment correlations. 


\section{Materials and methods}

\section{The experiment}

The potential effect of common environment on the degree of resemblance between parents and offspring was studied by means of a foster parent experiment (e.g. Dhondt, 1982). The rationale behind this type of experiment is that by exchanging clutches (or 2-day-old nestlings) between randomly chosen nests, one will break up the environmental correlation between offspring and their true parents. By regressing the trait values of offspring reared in randomly chosen foster nests against the values of their true parents, one will obtain heritability estimates $\left(h^{2}=\right.$ slope $)$ free from environmental correlation between offspring and parents. Thus, throughout, only offspring raised in foster nests were used in analyses. If these estimates do not differ from heritability estimates derived from analysis of nonfostered young, genotype-environment correlation may be judged not to have a large influence on heritability estimates. An additional insight into the importance of environmental determination of trait values was gained by regressing trait values of fostered young against the trait values of their unrelated guardians, i.e. foster parents. If fostered offspring converge in their trait values towards their foster parents, this will indicate non genetic causes of resemblance and call for caution in the interpretation of heritability estimates in general.

\section{The data}

Data were collected from 1983 to 1990 on the island of Gotland in the Baltic Sea $\left(57^{\circ} 10^{\prime} \mathrm{N}, 18^{\circ} 20^{\prime} \mathrm{E}\right)$, where a population of collared flycatchers has been intensively studied since 1980 (Gustafsson, 1985, 1990; Pärt, 1991). The family relationships between offspring and parents were determined by capturing parents in nest-boxes while they were feeding their young or incubating eggs (females). The mothers of young were known with certainty but because of extrapair fertilizations (Gelter et al., 1992), some inaccuracy may be involved in our estimates of father-offspring relationships. All fledglings were marked with numbered aluminum rings when 6-13 days old, which enabled individual recognition of birds recruited to the population in subsequent years. Heritabilities could then be estimated from mid-offspring-mid-parent regressions (Falconer, 1989; Merilä \& Gustafsson, 1993 ) by using measurements taken from fully or nearly fully grown (see below) offspring recruited to the population as adults ( 1 year old or more). Seven morphological traits were considered: wing length, first primary length, tail length, tarsus length, beak length, beak width and beak depth (all in $\mathrm{mm}$ ). The feather lengths were taken with a ruler to an accuracy of 0.5 $\mathrm{mm}$ and skeletal measures with slide or digital calipers to $0.1 \mathrm{~mm}$. The three feather lengths were first corrected for sex and age differences (see Merilä \& Gustafsson, 1993), because the age at which offspring and parents were measured often differed within and between families. Merilä \& Gustafsson (1993) give a more detailed account of measurement techniques.

Egg clutches or broods of 2-day-old nestlings were exchanged beween opportunistically chosen nests (same breeding phenology) located from $100 \mathrm{~m}$ to $2000 \mathrm{~m}$ apart. Transplants were made from 1983 to 1990 and produced 104 recruits deriving from 82 pairs of nests in which both true and foster parents had been measured for one or more traits. The number of offspring recruited from a family ranged from one $(83$ per cent) to three ( 5 per cent). In some cases the clutch/ brood size was increased or decreased with one or at most two eggs/young. As we know that heritability estimates are deflated when brood size is increased with two young, while no detectable effect is induced at other manipulation intensities $(-2,-1,+1$; $\mathrm{L}$. Gustafsson et al., unpublished data), we performed all analyses both excluding and including families in which brood size had been increased with two young. This did not have any qualitative effect on our results, probably because only two to five families (depending on trait) were affected.

\section{Results}

Heritability estimates of seven morphological traits obtained using fostered young are shown in Table 1 . There is no evidence of a consistent decrease in the estimates of heritability $\left(h^{2}\right)$ for fostered progeny against their natural parents compared with estimates obtained from unmanipulated broods (Table 1). Two of these $h^{2}$-estimates (beak and first primary length) are, however, considerably lower among foster offspring but none is significantly different from the estimate obtained by using unmanipulated broods (in all comparisons the 95 per cent confidence intervals overlap).

There was no resemblance between young and their foster parents (Table 1, Fig. 1). On the contrary, there was a tendency for negative foster parent-offspring regressions (Table 1). This clearly indicates that the resemblance between offspring and their natural parents is not caused by shared environment because in such a situation morphology of fostered young should converge towards that of their foster parents. In the absence of common environmental effects, foster parent regressions are expected to be zero and a change should give as many positive as negative regression slopes. The present result of six negative regres- 
Table 1 Heritability estimates $\left(h^{2}\right)$ from regressions of offspring on true parents and foster parents for seven morphological traits in fostered and natural broods of the collared flycatcher

\begin{tabular}{|c|c|c|c|c|c|c|c|c|}
\hline \multirow[b]{3}{*}{ Trait } & \multirow[b]{3}{*}{$N$} & \multicolumn{4}{|c|}{ Experimental } & \multirow{2}{*}{\multicolumn{3}{|c|}{$\frac{\text { Natural }}{\text { True parent }^{\mathrm{a}}}$}} \\
\hline & & \multicolumn{2}{|c|}{ Foster parent } & \multicolumn{2}{|c|}{ True parent } & & & \\
\hline & & $h^{2}$ & $\mathrm{SE}$ & $h^{2}$ & SE & $h^{2}$ & SE & $N$ \\
\hline Beak width & 40 & -0.22 & 0.18 & $0.48 * *$ & 0.16 & $0.40^{* * *}$ & 0.07 & 300 \\
\hline Beak depth & 38 & -0.32 & 0.33 & $0.56^{*}$ & 0.27 & $0.38^{* * *}$ & 0.07 & 298 \\
\hline Beak length & 59 & $-0.32^{\circ}$ & 0.18 & $0.35^{\circ}$ & 0.19 & $0.41 * * *$ & 0.05 & 681 \\
\hline Tarsus length & 59 & $-0.28^{\circ}$ & 0.15 & $0.59^{* * *}$ & 0.16 & $0.53^{* * *}$ & 0.04 & 691 \\
\hline Wing length & 61 & -0.02 & 0.16 & $0.60^{* * *}$ & 0.15 & $0.51^{* * *}$ & 0.05 & 681 \\
\hline Tail length & 59 & 0.01 & 0.17 & $0.41^{* *}$ & 0.16 & $0.45^{* * *}$ & 0.05 & 611 \\
\hline Primary length & 60 & -0.21 & 0.14 & 0.19 & 0.16 & $0.54 * * *$ & 0.05 & 585 \\
\hline Mean & & -0.19 & & 0.45 & & 0.46 & & \\
\hline
\end{tabular}

a Adapted from Merilä \& Gustafsson (1993).

All estimates are based on mid-offspring and mid-parent values.

$N=$ number of families.

Significance: ${ }^{\circ} P \leqslant 0.10,{ }^{*} P \leqslant 0.05,{ }^{* *} P \leqslant 0.01,{ }^{* * *} P \leqslant 0.001$
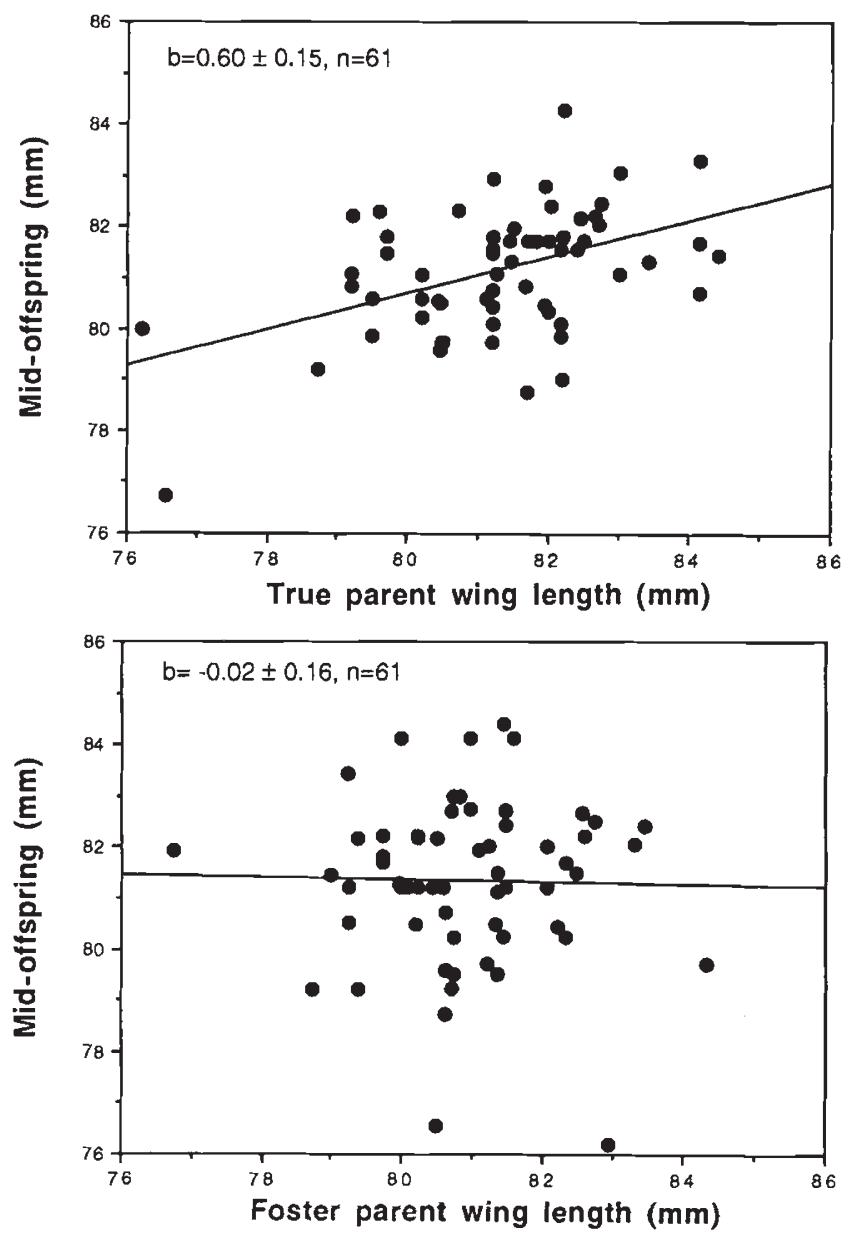

Fig. 1 Mean wing length of true (above) and foster parents (below) plotted against mean wing length of fostered collared flycatcher offspring.
Table 2 Regression coefficients $(b \pm S E)$ from the multiple regression of mid-offspring values on mid-foster parent and mid-true parent values

\begin{tabular}{|c|c|c|c|c|c|}
\hline \multirow[b]{2}{*}{ Trait } & \multirow[b]{2}{*}{$N$} & \multicolumn{2}{|c|}{ Foster parent } & \multicolumn{2}{|c|}{ True parent } \\
\hline & & $b$ & SE & $b$ & $\mathrm{SE}$ \\
\hline Beak width & 40 & -0.16 & 0.16 & $0.46^{* *}$ & 0.16 \\
\hline Beak depth & 39 & -0.45 & 0.32 & $0.46^{*}$ & 0.23 \\
\hline Beak length & 59 & $-0.44 *$ & 0.19 & $0.46^{*}$ & 0.20 \\
\hline Tarsus length & 59 & $-0.25^{\circ}$ & 0.15 & $0.56^{* * *}$ & 0.15 \\
\hline Wing length & 61 & -0.15 & 0.14 & $0.63^{* * *}$ & 0.15 \\
\hline Tail length & 59 & -0.12 & 0.17 & $0.45^{* *}$ & 0.18 \\
\hline Primary length & 60 & $-0.28^{\circ}$ & 0.16 & 0.17 & 0.18 \\
\hline
\end{tabular}

Significance: ${ }^{\circ} P \leqslant 0.10,{ }^{*} P \leqslant 0.05,{ }^{* *} P \leqslant 0.01,{ }^{* * *} P \leqslant 0.001$.

sions out of seven does not deviate from this expectation (binomial test, $P<0.098$, two-tailed).

The negative relationship between offspring and foster parent values is further illustrated by examining environmental deviations in offspring tarsus length, i.e. residuals from the regression of offspring on their true parents (Table 2). In all traits, environmental deviations bear a negative relationship to foster parent size, i.e. small foster parent values are associated with positive deviations in offspring values and vice versa. However, only the regression of beak length is significant $(P<0.05)$, while regressions of first primary and tarsus come close $(P<0.10)$. Beak length and length of first primary are the two traits that showed the largest 
difference between heritability estimates obtained using fostered and non fostered offspring (Table 1).

\section{Discussion}

An increasing number of studies (see Boag \& Noordwijk, 1987 for a review; Gustafsson, 1986; Alatalo \& Gustafsson, 1988; Lessells \& Ovenden, 1989; Payne \& Payne, 1989; Wiggins, 1989; Larsson \& Forslund, 1992; Merilä \& Gustafsson, 1993) have estimated heritabilities of morphological traits in natural populations of birds. However, only a few of these studies have controlled for genotype-environment correlations (Smith \& Dhondt, 1980; Dhondt, 1982; Alatalo \& Lundberg, 1986; Alatalo \& Gustafsson, 1988; Wiggins, 1989), even though such correlations are known to occur (James, 1983; James \& NeSmith, 1988; Rhymer, 1992). The existence of genotype-environment correlation precludes the valid estimation of genotypic, environmental and interaction contributions to the phenotypic variance because in its presence it is impossible to tell how much of the phenotypic similarity among relatives is due to shared genes and how much to shared environment (Feldman \& Lewontin, 1975; Falconer, 1989).

Our results demonstrate that heritabilities estimated using offspring raised by unrelated foster parents did not differ significantly from heritabilities estimated from natural broods (Merilä \& Gustafsson, 1993). Thus, resemblance between offspring and parents is neither caused, nor even inflated, by environmental correlation between them (Table 1). When there are strong environmental effects, shared environment should also increase the similarity between fostered young and their foster parents but no such tendency was found in this study.

However, the heritability estimates for beak and first primary length were not significant, which could indicate that similarity of these traits is inflated by environmental correlation between parents and offspring. However, as no resemblance was found between foster parents and offspring, this seems unlikely. Both these traits are measured with some error and they grow and wear continuously (repeatabilities 56 per cent and 67 per cent for first primary and beak length, respectively; Merilä \& Gustafsson, 1993). Since this inaccuracy, in combination with relatively small sample sizes, may yield nonsignificant regression slopes, it is not justifiable to take the lack of significance as a proof for strong non genetic effects, especially in the absence of similarity between foster parents and foster offspring.

Surprisingly, there was a clear tendency for negative offspring-foster parent regressions. A negative correla- tion between offspring and foster parent values could arise simply if young from large parents were fostered by small foster parents and vice versa. This, however, was not the case: the correlations between true and foster parent values were always positive and significantly so in beak $\left(r_{s}=0.26\right)$, tail $\left(r_{s}=0.30\right)$ and first primary $\left(r_{s}=0.48\right)$ length (in all, $\left.P<0.05\right)$. An alternative possibility is that foster parents actually affected the phenotype of fostered young. As illustrated in Table 2, even after controlling for genetic transmission between offspring and true parents, the effects of foster parent size are significant for beak length and nearly so for first primary and tarsus length. This indicates that foster parents indeed affected the environmental deviations in offspring size and that the sign and magnitude of this effect was proportional to their own size. That the two lowest true parent regressions (Table 1) were among those in which environmental deviations in offspring size might have been affected by foster parent size may suggest causality between low $h^{2}$-estimates and negative offspring-foster parent regressions. A possible proximate mechanism creating this negative correlation between size of fostered young and their foster parents is that some fostered young grew up in experimentally increased broods (see Materials and methods), which equate to inferior environments. If large foster parents had difficulty in raising young to an equivalent size as do small foster parents in deteriorated environments, a negative relationship between offspring and parental size might be expected. On the other hand, this negative relationship is not expected to occur in good environments. We checked this possibility by using a larger sample of foster young measured for tarsus length. Since tarsus length attains its final size before the young fledge, this analysis allows us to use a larger data set which is not dependent on offspring recruiting to the population. Figure 2 shows that the tarsus length of fostered young is negatively related to that of their foster parents in increased broods $(P<0.02)$ but not in control broods $(P>0.05)$. Furthermore, the interaction term between foster parent tarsus and the environment (enlarged vs. control $)$ is highly significant $\left(F_{2,142}=5.0, P<0.01\right)$. Thus, this evidence is in concordance with the results presented above and indicates that the negative relationship between offspring and their foster parent size is real, giving support to the hypothesis that large foster parents have more difficulties in raising their offspring under poor environmental conditions than do the smaller foster parents. Why this should be the case is unclear and the question falls outside the scope of this paper.

In conclusion, our results argue that the observed phenotypic variation in collared flycatcher morphology 

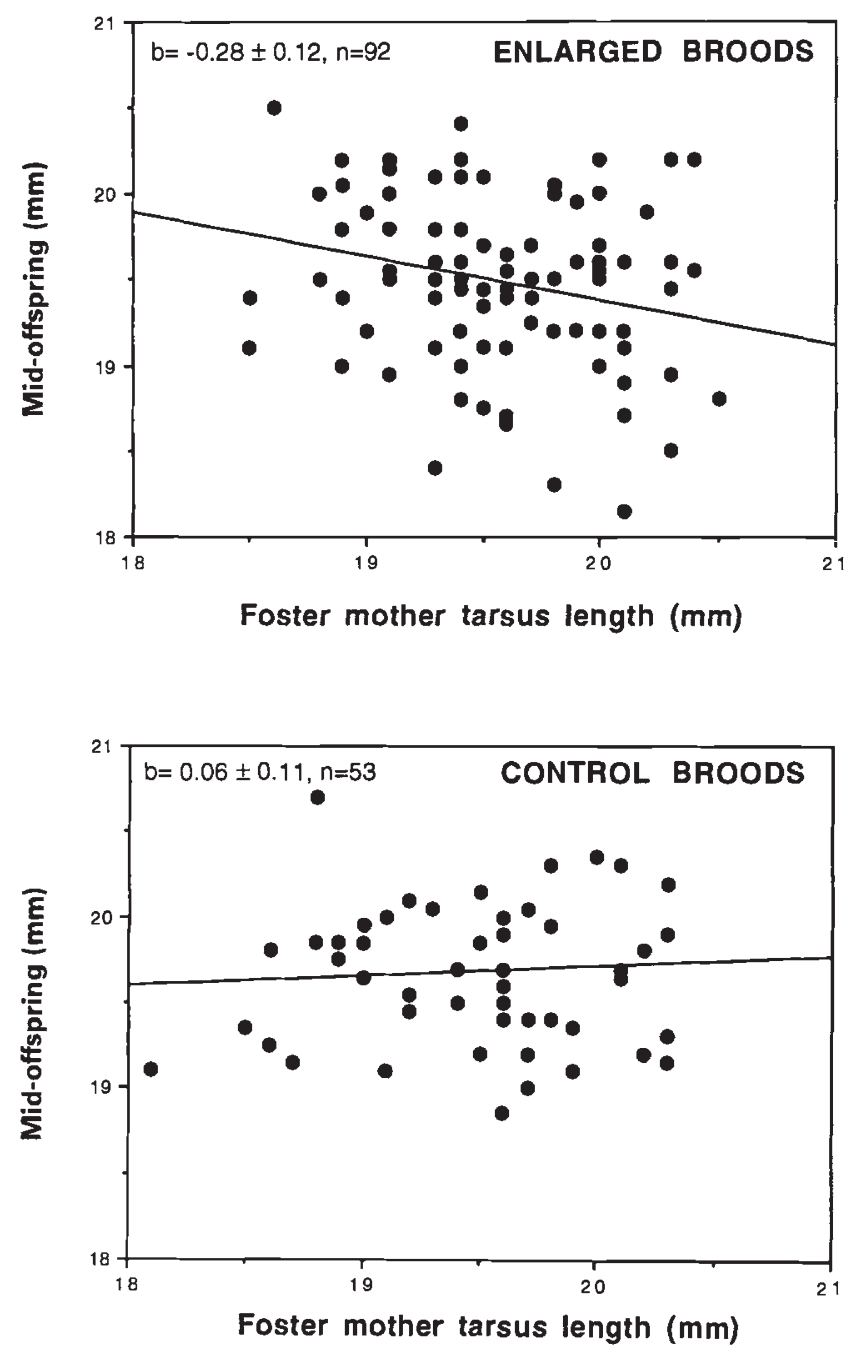

Fig. 2 Foster offspring tarsus length in relation to foster mother tarsus length in enlarged (above; $F_{1,90}=5.64$, $P<0.02$ ) and control broods (below, $F_{1,51}=0.29, P=0.59$ ).

truly reflects additive genetic variation. Our experiment failed to reveal any contribution to heritability estimates from the rearing environment, despite the fact that environmental variation has been demonstrated to influence size of the young in this population (Gustafsson, 1988, 1990; Alatalo et al., 1990). Our results provide the first experimental evidence for a heritable basis of phenotypic variation in feather traits and suggest that collared flycatcher morphology could respond rapidly to directional selection.

\section{Acknowledgements}

We thank Staffan Ulfstrand and one anonymous reviewer who read the manuscript and made several suggestions that improved it. This study was supported by grants from the Swedish Natural Science Research
Council to L.G. and by grants from The Royal Swedish Academy of Sciences (P. F. Wahlberg's minnesfond, Hierta-Retzius fond) to J.M.

\section{References}

ALATALO, R. V. AND GUSTAFSSON, L. 1988. Genetic component of morphological differentiation in coal tits under competitive release. Evolution, 42, 200-203.

Alatalo, R. V., Gustafsson, L. AND LundBerg, A. 1990. Phenotypic selection on heritable size traits: environmental variance and genetic response. Am. Nat., 135, 464-471.

ALATALO, R. V. AND LundBerG, A. 1986. Heritability and selection on tarsus length in the pied flycatcher (Ficedula hypoleuca). Evolution, 45, 574-583.

BOAG, P. T. AND VAN NOORDWIJK, A. J. 1987. Quantitative Genetics. In: Cooke, F. and Buckley, P. A. (eds) Avian Genetics, pp. 45-77. Academic Press, London.

DHONDT, A. 1982. Heritability of blue tit tarsus length from normal and cross-fostered broods. Evolution, 36, 418-419.

FALCONER, D. S. 1989. Introduction to Quantitative Genetics, 3rd edn. Longman, London.

FELDMAN, M. W. AND LEWONTIN, R. C. 1975. The heritability hang-up. Science, 190, 1163-1168.

GELTER, H. P., TEgelströM, H. AND GUSTAFSSON, L. 1992. Evidence from hatching success and DNA fingerprinting for the fertility of hybrid Pied $\times$ Collared flycatchers Ficedula hypoleuca $\times$ albicollis. Ibis, 134, 62-68.

GUSTAFSSON, L. 1985. Fitness factors in the collared flycatcher, Ficedula albicollis Temm. Ph.D. Thesis, Uppsala University, Sweden.

GUSTAFSSON, L. 1986. Lifetime reproductive success and heritability: empirical support for Fisher's fundamental theorem. Am. Nat., 128, 761-764.

GUSTAFSSON, L. 1988. Interspecific competition lowers fitness in the collared flycatcher (Ficedula albicollis). Ecology, 68, 281-286.

GUSTAFSSON, L. 1990. Life-history trade-offs and optimal clutch size in relation to age in the Collared Flycatcher. In: Blondel, J., Gosler, A., Lebreton, J.-D. and McCleery, R. (eds) Population Biology of Passerine Birds - an Integrated Approach, pp. 235-246. NATO ASI Series G, Ecological Sciences, Springer, Berlin.

JAMES, F. C. 1983. Environmental component of morphological differentiation in birds. Science, 221, 184-186.

JAMES, F. C. AND NESMITH, C. 1988. Non-genetic effects in geographical differences among nestling populations of Red-winged Blackbirds. In: Quellet, H. (ed.) Acta XIX Congressus Internationalis Ornithologici, vol. II, pp. 1424-1433, Ottawa, Canada.

LARSSON, K. AND FORSLUND, P. 1992. Genetic and social inheritance of body and egg size in the barnacle goose (Branta leucopsis). Evolution, 46, 235-244.

LESSELS, C. M. AND OVENDEN, G. N. 1989. Heritability of wing length and weight in European bee-eaters (Merops apiaster). Condor, 91, 210-214. 
MERILÄ, J. AND GUSTAFSSON, L. 1993. Inheritance of size and shape in a natural population of collared flycatchers, Ficedula albicollis. J. Evol. Biol., 6, 375-395.

PAYNE, R. B. AND PAYNE, L. L. 1989. Heritability estimates and behaviour observations: extra-pair matings in indigo buntings. Anim. Behav., 38, 457-467.

PÅRT, T. 1991. Philopatry and age as factors influencing reproductive success in the collared flycatcher (Ficedula albicollis). Ph.D. Thesis, University of Uppsala, Sweden.

RAUSHER, M. D. 1992. The measurement of selection on quantitative traits: biases due to environmental covariances between traits and fitness. Evolution, 46, 616-626.

RHYMER, J. M. 1992. An experimental study of geographic variation in avian growth and development. J. Evol. Biol., 5, 289-306.
SMITH, J. N. AND DHONDT, A. 1980. Experimental confirmation of heritable morphological variation in a natural population of song sparrows. Evolution, 34, 1155-1158.

STEARNS, s. C. 1992. The Evolution of Life Histories. Oxford University Press, Oxford.

TURNER, H. N. AND YOUNG, s. Y. 1969. Quantitative Genetics in Sheep Breeding. Cornell University Press, Ithaca, New York.

VAN NOORDWIJK, A. J., VAN BALEN, J. H. AND SCHARLOO, w. 1988. Heritability of body size in a natural population of the great tit (Parus major) and its relation to age and environmental conditions during growth. Genet. Res., 51, 149-162.

WIGGINS, D. A. 1989. Heritability of body size in cross-fostered tree swallow broods. Evolution, 43, 1808-1811. 